\title{
On a Risk Model with Surplus-dependent Premium and Tax Rates
}

\author{
Eric C. K. Cheung • David Landriault
}

Received: 1 December 2009 / Revised: 19 July 2010 /

Accepted: 15 September 2010 / Published online: 21 October 2010

(C) The Author(s) 2010. This article is published with open access at Springerlink.com

\begin{abstract}
In this paper, the compound Poisson risk model with surplus-dependent premium rate is analyzed in the taxation system proposed by Albrecher and Hipp (Blätter der DGVFM 28(1):13-28, 2007). In the compound Poisson risk model, Albrecher and Hipp (Blätter der DGVFM 28(1):13-28, 2007) showed that a simple relationship between the ruin probabilities in the risk model with and without tax exists. This so-called tax identity was later generalized to a surplus-dependent tax rate by Albrecher et al. (Insur Math Econ 44(2):304-306, 2009). The present paper further generalizes these results to the Gerber-Shiu function with a generalized penalty function involving the maximum surplus prior to ruin. We show that this generalized Gerber-Shiu function in the risk model with tax is closely related to the 'original' Gerber-Shiu function in the risk model without tax defined in a dividend barrier framework. The moments of the discounted tax payments before ruin and the optimal threshold level for the tax authority to start collecting tax payments are also examined.
\end{abstract}

Keywords Gerber-Shiu function - Tax identity • Maximum surplus level • Surplus-dependent premium $\cdot$ Discounted tax payments

AMS 2000 Subject Classifications Primary 91B30; Secondary 60G55 • 60J75 • 90B05

E. C. K. Cheung

Department of Statistics and Actuarial Science,

University of Hong Kong, Pokfulam, Hong Kong

D. Landriault $(\bowtie)$

Department of Statistics and Actuarial Science,

University of Waterloo, 200 University Avenue West, Waterloo, Canada

e-mail: dlandriault@uwaterloo.ca 


\section{Introduction}

In this paper, we consider the compound Poisson risk model with surplus-dependent premium rate. Let $\{S(t)\}_{t \geq 0}$ be the aggregate claim amount process defined as

$$
S(t)=\left\{\begin{array}{ll}
\sum_{i=1}^{N(t)} Y_{i}, & N(t)>0 \\
0, & N(t)=0
\end{array},\right.
$$

where $\{N(t)\}_{t \geq 0}$ is a Poisson process with rate $\lambda>0$ and $\left\{Y_{i}\right\}_{i=1}^{\infty}$ is a sequence of independent and identically distributed (i.i.d.) positive random variables with density $p\left(\right.$.) and finite mean. We assume that the claim number process $\{N(t)\}_{t \geq 0}$ and the claim sizes $\left\{Y_{i}\right\}_{i=1}^{\infty}$ are independent.

Let $U(t)$ be the surplus level at time $t \geq 0$. It is assumed that the insurer's instantaneous premium rate at time $t$ is $c(U(t))$ where $c($.$) is a positive deterministic$ function. The motivation for a surplus-dependent premium rate is two-fold. First, as argued by Lin and Pavlova (2006), a large surplus level allows the insurer to reduce premiums in order to stay competitive. On the other hand, when the surplus level runs low, the insurer is likely to charge a higher premium to avoid insufficiency of funds. Second, from a mathematical viewpoint, the class of risk models with surplus-dependent premium rate includes a large variety of risk models involving dividend strategies and/or interest earnings as special cases (see Section 2.3 for some examples).

The surplus process $\{U(t)\}_{t \geq 0}$ is defined via the dynamics

$$
d U(t)=c(U(t)) d t-d S(t), \quad t \geq 0,
$$

with $U(0)=u$. In this paper, the compound Poisson risk model modified by tax payments under a loss-carry forward tax system is studied. Such a tax model was first proposed by Albrecher and Hipp (2007) in the context of a constant tax rate and was later generalized by Albrecher et al. (2009) to a surplus-dependent tax rate. Under this taxation system, it is assumed that, whenever the surplus process $\left\{U_{\Gamma}(t)\right\}_{t \geq 0}$ is at its running maximum $\bar{U}_{\Gamma}(t)=\sup _{0 \leq s \leq t} U_{\Gamma}(s)$ at a given time $t \geq 0$, a fraction $\gamma\left(U_{\Gamma}(t)\right)$ of the premium income is paid as tax and as a result, the surplus process grows at the reduced rate $c\left(U_{\Gamma}(t)\right)\left[1-\gamma\left(U_{\Gamma}(t)\right)\right]$. Hence, the surplus process $\left\{U_{\Gamma}(t)\right\}_{t \geq 0}$ under the above tax system satisfies

$$
d U_{\Gamma}(t)=c\left(U_{\Gamma}(t)\right)\left[1-\gamma\left(\bar{U}_{\Gamma}(t)\right) I\left(U_{\Gamma}(t)=\bar{U}_{\Gamma}(t)\right)\right] d t-d S(t), \quad t \geq 0,
$$

where $I(A)$ is the indicator function of the event $A$. Throughout the paper, we assume that the tax function $\gamma($.) satisfies $0 \leq \gamma(x)<1$ for $x \geq 0$. Clearly, when $\gamma(.) \equiv 0$ (i.e. in a tax-free environment), the surplus process $\left\{U_{\Gamma}(t)\right\}_{t \geq 0}$ reduces to $\{U(t)\}_{t \geq 0}$.

Remark 1 Note that the tax rate $\gamma($.) is a function of the surplus level of the process $\left\{U_{\Gamma}(t)\right\}_{t \geq 0}$ with tax (e.g. Albrecher et al. 2009) as opposed to Kyprianou and Zhou (2009) and Renaud (2009) where the tax rate is a function of the surplus level of the 
process $\{U(t)\}_{t \geq 0}$ without tax. As shown by Kyprianou and Zhou (2009, Lemma 1), whenever the premium rate is constant (i.e. $c(.) \equiv c$ ), the processes $\{U(t)\}_{t \geq 0}$ and $\left\{U_{\Gamma}(t)\right\}_{t \geq 0}$ attain their respective running maximums at the same time and there is a one-to-one correspondence between the two running maximums. However, this is generally not true when the premium rate is surplus-dependent. Therefore, we have chosen the former definition which is more natural.

The time of ruin $T_{\Gamma}$ of the surplus process $\left\{U_{\Gamma}(t)\right\}_{t \geq 0}$ is defined as $T_{\Gamma}=\inf \{t \geq 0$ : $\left.U_{\Gamma}(t)<0\right\}$ where $T_{\Gamma}=\infty$ if ruin does not occur. Its ruin probability $\psi_{\Gamma}(u)$ is given by $\psi_{\Gamma}(u)=\operatorname{Pr}\left\{T_{\Gamma}<\infty \mid U_{\Gamma}(0)=u\right\}$. With a slight abuse of notation, in the remainder of this paper the subscript ' $\Gamma$ ' is replaced by ' $\gamma$ ' whenever the tax rate is constant (i.e. $\gamma(.) \equiv \gamma)$. Similarly, in the absence of tax payments, we further replace the subscript ' $\gamma$ ' by ' 0 '. For instance, $\psi_{\gamma}(u)$ and $\psi_{0}(u)$ are the ruin probabilities for the process (1.2) with $\gamma(.) \equiv \gamma$ and $\gamma(.) \equiv 0$ respectively. If $\gamma($.$) is bounded by a constant$ less than 1 , then the condition $\psi_{0}(0)<1$ is sufficient to guarantee that $\psi_{\Gamma}(u)<1$ for $\forall u \geq 0$ (see Albrecher et al. 2009). In the sequel, we also require that the following two technical conditions are fulfilled, namely

$$
\int_{0}^{x} \frac{d z}{c(z)[1-\gamma(z)]}<\infty \text { for any finite } x \geq 0 \quad \text { and } \quad \int_{0}^{\infty} \frac{d z}{c(z)}=\infty
$$

See Lin and Sendova (2008).

For the case of a constant premium rate $c(.) \equiv c$, Albrecher et al. (2009) showed that

$$
1-\psi_{\Gamma}(u)=\exp \left\{-\int_{u}^{\infty} \frac{1}{1-\gamma(x)} \frac{d}{d x} \ln \left[1-\psi_{0}(x)\right] d x\right\}, \quad u \geq 0,
$$

which is an extension of the so-called tax identity

$$
1-\psi_{\gamma}(u)=\left[1-\psi_{0}(u)\right]^{\frac{1}{1-\gamma}}, \quad u \geq 0,
$$

first established by Albrecher and Hipp (2007). Ruin probabilities and other ruinrelated quantities (e.g. the total discounted tax payments before ruin) have since been analyzed in other risk models. For instance, Albrecher et al. (2008a) showed that the tax identity (1.5) with minor adjustments remains valid in a dual risk model with exponentially distributed innovations. Wei (2009) examined a compound Poisson risk model with credit interest and showed that Eq. 1.5 also holds. Albrecher et al. (2008b) and Kyprianou and Zhou (2009) showed that the identities (1.5) and (1.4) remain valid for a Lévy insurance risk model with constant tax rate and general tax structure respectively. In this paper, we notably extend the identity (1.4) to a generalization of the Gerber-Shiu discounted penalty function.

For the surplus process $\left\{U_{\Gamma}(t)\right\}_{t \geq 0}$, the 'original' Gerber-Shiu discounted penalty function $m_{\Gamma}(u)$ is defined as

$$
m_{\Gamma}(u)=E\left[e^{-\delta T_{\Gamma}} w\left(U_{\Gamma}\left(T_{\Gamma}^{-}\right),\left|U_{\Gamma}\left(T_{\Gamma}\right)\right|\right) I\left(T_{\Gamma}<\infty\right) \mid U_{\Gamma}(0)=u\right], \quad u \geq 0,
$$

where $\delta \geq 0$ is the force of interest or a Laplace transform argument, and $w: \mathbb{R}^{2} \rightarrow \mathbb{R}$ is the so-called penalty function depending on the surplus prior to ruin $U_{\Gamma}\left(T_{\Gamma}^{-}\right)$and 
the deficit at ruin $\left|U_{\Gamma}\left(T_{\Gamma}\right)\right|$ (see Gerber and Shiu 1998). We remark that when both the tax rate and premium rate are constant, the quantity $m_{\gamma}(u)$ was studied by Ming et al. (2010) under an 'absolute ruin' setting (see e.g. Cai 2007 for the definition of 'absolute ruin'). In this paper, we extend Eq. 1.6 by further incorporating the maximum surplus level before ruin (or equivalently the running maximum at the time of ruin), namely $\bar{U}_{\Gamma}\left(T_{\Gamma}\right)$ into the analysis. As in Cheung and Landriault (2010), we consider the generalized analytic tool

$$
\phi_{\Gamma}(u)=E\left[e^{-\delta T_{\Gamma}} w\left(U_{\Gamma}\left(T_{\Gamma}^{-}\right),\left|U_{\Gamma}\left(T_{\Gamma}\right)\right|\right) w_{*}\left(\bar{U}_{\Gamma}\left(T_{\Gamma}\right)\right) I\left(T_{\Gamma}<\infty\right) \mid U_{\Gamma}(0)=u\right], \quad u \geq 0,
$$

with $w_{*}$ (.) a real-valued function. Clearly, the Gerber-Shiu function $m_{\Gamma}(u)$ in Eq. 1.6 is a special case of $\phi_{\Gamma}(u)$ in Eq. 1.7 with $w_{*}(.) \equiv 1$.

We point out that the multiplicative form of the penalty function $w(.,.) w_{*}($.$) is not$ as restrictive as one may think. For instance, the discounted joint density of the triplet $\left(U_{\Gamma}\left(T_{\Gamma}^{-}\right),\left|U_{\Gamma}\left(T_{\Gamma}\right)\right|, \bar{U}_{\Gamma}\left(T_{\Gamma}\right)\right)$ can be analyzed through Eq. 1.7 with $w(x, y)=e^{-q x-r y}$ and $w_{*}(z)=e^{-s z}$ (see Cheung and Landriault 2010, Section 4.2). Also, the density function of the distance between the running maximum and running minimum of $\left\{U_{\Gamma}(t)\right\}_{t \geq 0}$ at the time of ruin, namely $\bar{U}_{\Gamma}\left(T_{\Gamma}\right)+\left|U_{\Gamma}\left(T_{\Gamma}\right)\right|$, can also be studied via the Gerber-Shiu function $\phi_{\Gamma}(u)$ (see Cheung and Landriault 2010, Proposition 1). The analysis of an additive penalty function of the form $w(.,)+.w_{*}($.$) is also possible via$ the sum of two Gerber-Shiu functions of the form (1.7).

Under the aforementioned tax framework, we also examine the $n$-th moment of discounted tax payments paid until ruin, namely

$$
\begin{aligned}
W_{n, \Gamma}(u)= & E\left[\left\{\int_{0}^{T_{\Gamma}} e^{-\delta v} \gamma\left(U_{\Gamma}(v)\right) c\left(U_{\Gamma}(v)\right)\right.\right. \\
& \left.\left.\times I\left(U_{\Gamma}(v)=\bar{U}_{\Gamma}(v)\right) d v\right\}^{n} \mid U_{\Gamma}(0)=u\right], \quad u \geq 0,
\end{aligned}
$$

for $n=0,1,2, \ldots$, with the usual convention that $W_{0, \Gamma}(.) \equiv 1$. As far as the discounted tax payment is concerned, we always assume $\delta>0$. While the quantity $W_{1, \gamma}(u)$ was studied by Albrecher and Hipp (2007) when $c(.) \equiv c$, an analysis of the higher moments $\left\{W_{n, \gamma}(u)\right\}_{n=1}^{\infty}$ in the context of a Lévy insurance risk model can be found in Albrecher et al. (2008b). We also refer interested readers to Kyprianou and Zhou (2009) and Renaud (2009) respectively for the study of $W_{1, \Gamma}(u)$ and $\left\{W_{n, \Gamma}(u)\right\}_{n=1}^{\infty}$ in the Lévy insurance risk model with general tax structure.

This paper is organized as follows. In Section 2, an expression for the GerberShiu function $\phi_{\Gamma}(u)$ in terms of the Laplace transform of a first passage time and the 'original' Gerber-Shiu function in a risk model with a dividend barrier strategy is derived. The moments of the discounted tax payments are the subject matter of Section 3. Section 4 considers the situation where tax payments only start when the surplus process reaches a given threshold level. The optimal threshold level which maximizes the difference between the expected value of total discounted tax payments and the 'original' Gerber-Shiu function is also examined. Section 5 ends the paper by some concluding remarks. 


\section{Analysis of the Generalized Gerber-Shiu Function}

\subsection{A Differential Equation}

Up to the time of the first claim, the surplus process $\left\{U_{\Gamma}(t)\right\}_{t \geq 0}$ is at its running maximum. For a first claim occurring at time $t$, the surplus level just before the payment of this claim is

$$
f_{\Gamma}(u, t)=u+\int_{0}^{t} c\left(f_{\Gamma}(u, v)\right)\left[1-\gamma\left(f_{\Gamma}(u, v)\right)\right] d v .
$$

If the first claim does not cause ruin, there are two possibilities as the process further evolves:

- the surplus process reaches its running maximum $f_{\Gamma}(u, t)$ before ruin, or

- the surplus process does not reach its running maximum $f_{\Gamma}(u, t)$ before ruin, and hence the maximum surplus prior to ruin is the surplus level just prior to the first claim.

On the other hand, if the first claim causes ruin, the maximum surplus level prior to ruin is identical to the surplus prior to ruin. Combining the above cases, one finds that

$$
\begin{aligned}
\phi_{\Gamma}(u)= & \int_{0}^{\infty} \lambda e^{-(\lambda+\delta) t}\left[\int_{0}^{f_{\Gamma}(u, t)} \chi_{\delta}\left(f_{\Gamma}(u, t)-y ; f_{\Gamma}(u, t)\right) p(y) d y\right] \phi_{\Gamma}\left(f_{\Gamma}(u, t)\right) d t \\
& +\int_{0}^{\infty} \lambda e^{-(\lambda+\delta) t}\left[\int_{0}^{f_{\Gamma}(u, t)} l\left(f_{\Gamma}(u, t)-y ; f_{\Gamma}(u, t)\right) p(y) d y\right] w_{*}\left(f_{\Gamma}(u, t)\right) d t \\
& +\int_{0}^{\infty} \lambda e^{-(\lambda+\delta) t}\left[\int_{f_{\Gamma}(u, t)}^{\infty} w\left(f_{\Gamma}(u, t), y-f_{\Gamma}(u, t)\right) p(y) d y\right] w_{*}\left(f_{\Gamma}(u, t)\right) d t,
\end{aligned}
$$

for $u \geq 0$ where

$$
\chi_{\delta}(u ; b)=E\left[e^{-\delta \tau_{b}} I\left(\tau_{b}<T_{0}\right) \mid U_{0}(0)=u\right], \quad 0 \leq u \leq b,
$$

is the Laplace transform of the first passage time $\tau_{b}=\inf \left\{t \geq 0: U_{0}(t)=b\right\}$ avoiding ruin enroute in the model without tax, and

$$
l(u ; b)=E\left[e^{-\delta T_{0}} w\left(U_{0}\left(T_{0}^{-}\right),\left|U_{0}\left(T_{0}\right)\right|\right) I\left(T_{0}<\tau_{b}\right) \mid U_{0}(0)=u\right], \quad 0 \leq u \leq b,
$$

is the 'original' Gerber-Shiu function with the event that the surplus process does not upcross level $b$ before ruin in the model without tax. We remark that notationally we emphasize the dependence of the quantity $\chi_{\delta}(u ; b)$ on $\delta$, and this will be particularly useful in Section 3.

Changing the variable of integration from $t$ to $x=f_{\Gamma}(u, t)$ in Eq. 2.2, it follows from Eq. 2.1 that $d x=c\left(f_{\Gamma}(u, t)\right)\left[1-\gamma\left(f_{\Gamma}(u, t)\right)\right] d t$. This implies that $d t=$ $(c(x)[1-\gamma(x)])^{-1} d x$. Given that $x=u$ when $t=0$, we have $t=k_{\Gamma}(u, x)$ where

$$
k_{\Gamma}(u, x)=\int_{u}^{x} \frac{d z}{c(z)[1-\gamma(z)]}, \quad x \geq u .
$$


Note that the two technical conditions (Eq. 1.3) are necessary to ensure a proper definition of $k_{\Gamma}(u, x)$. It follows that

$$
\begin{aligned}
\phi_{\Gamma}(u)= & \int_{u}^{\infty} \frac{\lambda}{c(x)[1-\gamma(x)]} e^{-(\lambda+\delta) k_{\Gamma}(u, x)}\left[\int_{0}^{x} \chi_{\delta}(x-y ; x) p(y) d y\right] \phi_{\Gamma}(x) d x \\
& +\int_{u}^{\infty} \frac{\lambda}{c(x)[1-\gamma(x)]} e^{-(\lambda+\delta) k_{\Gamma}(u, x)}\left[\int_{0}^{x} l(x-y ; x) p(y) d y+\alpha(x)\right] w_{*}(x) d x,
\end{aligned}
$$

where

$$
\alpha(x)=\int_{x}^{\infty} w(x, y-x) p(y) d y, \quad x \geq 0 .
$$

Differentiating Eq. 2.6 with respect to (w.r.t.) $u$ leads to

$$
\begin{aligned}
\phi_{\Gamma}^{\prime}(u)= & \frac{\phi_{\Gamma}(u)}{c(u)[1-\gamma(u)]}\left[(\lambda+\delta)-\lambda \int_{0}^{u} \chi_{\delta}(u-y ; u) p(y) d y\right] \\
& -\frac{\lambda}{c(u)[1-\gamma(u)]}\left[\int_{0}^{u} l(u-y ; u) p(y) d y+\alpha(u)\right] w_{*}(u),
\end{aligned}
$$

for $u \geq 0$. Note that Eq. 2.7 is a first-order non-homogeneous differential equation with non-constant coefficients. We find the solution of the differential equation (2.7) by further analyzing the Laplace transform of the first passage time $\tau_{b}$, namely $\chi_{\delta}(u ; b)$.

\subsection{Analysis of $\chi_{\delta}(u ; b)$}

Recall that the Laplace transform $\chi_{\delta}(u ; b)$ pertains to the non-tax surplus process $\left\{U_{0}(t)\right\}_{t \geq 0}$. Thus, for $\gamma(.) \equiv 0$, Eq. 2.5 becomes

$$
k_{0}(u, x)=\int_{u}^{x} \frac{d z}{c(z)}, \quad x \geq u,
$$

where $k_{0}(u, x)$ corresponds to the time that $\left\{U_{0}(t)\right\}_{t \geq 0}$ reaches level $x$ (from level $u$ ) if no claim occurs enroute. By conditioning on the time and amount of the first claim, one finds that

$$
\chi_{\delta}(u ; b)=\int_{0}^{k_{0}(u, b)} \lambda e^{-(\lambda+\delta) t}\left[\int_{0}^{f_{0}(u, t)} \chi_{\delta}\left(f_{0}(u, t)-y ; b\right) p(y) d y\right] d t+e^{-(\lambda+\delta) k_{0}(u, b)},
$$

for $0 \leq u \leq b$. By a change of variable from $t$ to $x=f_{0}(u, t)$ in Eq. 2.8, one deduces that

$$
\chi_{\delta}(u ; b)=\int_{u}^{b} \frac{\lambda}{c(x)} e^{-(\lambda+\delta) k_{0}(u, x)}\left[\int_{0}^{x} \chi_{\delta}(x-y ; b) p(y) d y\right] d x+e^{-(\lambda+\delta) k_{0}(u, b)} .
$$


Differentiating Eq. 2.9 w.r.t. $u$ yields the homogeneous integro-differential equation

$$
\chi_{\delta}^{\prime}(u ; b)=\frac{\lambda+\delta}{c(u)} \chi_{\delta}(u ; b)-\frac{\lambda}{c(u)} \int_{0}^{u} \chi_{\delta}(u-y ; b) p(y) d y,
$$

for $0 \leq u \leq b$. Equation 2.10 together with the trivial boundary condition $\chi_{\delta}(b ; b)=1$ (see Gerber et al. 2006a) implies that

$$
\chi_{\delta}(u ; b)=\frac{v_{\delta}(u)}{v_{\delta}(b)}, \quad 0 \leq u \leq b,
$$

where $v_{\delta}(u)$ is a non-trivial solution of the homogeneous integro-differential equation

$$
v_{\delta}^{\prime}(u)=\frac{\lambda+\delta}{c(u)} v_{\delta}(u)-\frac{\lambda}{c(u)} \int_{0}^{u} v_{\delta}(u-y) p(y) d y, \quad u \geq 0,
$$

which is unique up to a multiplicative constant (see e.g. Lakshmikantham and Rao 1995). Without loss of generality, one may assume $v_{\delta}(0)=1$. From Eq. 2.11, it is clear that $v_{\delta}(u)$ is a non-decreasing function in $u$. In cases where the premium rate is constant, $v_{\delta}(u)$ is simply (a constant multiple of) the scale function in the literature of Lévy processes (see e.g. Biffis and Kyprianou 2010).

\subsection{Solution to the Generalized Gerber-Shiu Function}

Substituting Eq. 2.11 in Eq. 2.7 followed by an application of Eq. 2.12 leads to

$$
\begin{aligned}
\phi_{\Gamma}^{\prime}(u)= & \frac{\phi_{\Gamma}(u)}{c(u)[1-\gamma(u)]}\left[(\lambda+\delta)-\frac{\lambda}{v_{\delta}(u)} \int_{0}^{u} v_{\delta}(u-y) p(y) d y\right] \\
& -\frac{\lambda}{c(u)[1-\gamma(u)]}\left[\int_{0}^{u} l(u-y ; u) p(y) d y+\alpha(u)\right] w_{*}(u) \\
= & \frac{\phi_{\Gamma}(u)}{1-\gamma(u)} \frac{v_{\delta}^{\prime}(u)}{v_{\delta}(u)}-\frac{\lambda}{c(u)[1-\gamma(u)]}\left[\int_{0}^{u} l(u-y ; u) p(y) d y+\alpha(u)\right] w_{*}(u),
\end{aligned}
$$

for $u \geq 0$. Rearranging Eq. 2.13 gives rise to

$$
\phi_{\Gamma}^{\prime}(u)-\frac{1}{1-\gamma(u)} \frac{v_{\delta}^{\prime}(u)}{v_{\delta}(u)} \phi_{\Gamma}(u)=-\frac{\lambda}{c(u)[1-\gamma(u)]}\left[\int_{0}^{u} l(u-y ; u) p(y) d y+\alpha(u)\right] w_{*}(u) .
$$

Note that the above equation also holds true for the 'original' Gerber-Shiu function $m_{0}(u)$ without tax payments (by letting $w_{*}(.) \equiv 1$ and $\left.\gamma(.) \equiv 0\right)$, i.e.

$$
m_{0}^{\prime}(u)-\frac{v_{\delta}^{\prime}(u)}{v_{\delta}(u)} m_{0}(u)=-\frac{\lambda}{c(u)}\left[\int_{0}^{u} l(u-y ; u) p(y) d y+\alpha(u)\right] .
$$


Comparing Eqs. 2.14 and 2.15, one deduces that

$$
\phi_{\Gamma}^{\prime}(u)-\frac{1}{1-\gamma(u)} \frac{v_{\delta}^{\prime}(u)}{v_{\delta}(u)} \phi_{\Gamma}(u)=\frac{1}{1-\gamma(u)}\left[m_{0}^{\prime}(u)-\frac{v_{\delta}^{\prime}(u)}{v_{\delta}(u)} m_{0}(u)\right] w_{*}(u),
$$

for $u \geq 0$. By multiplying both sides of Eq. 2.16 by the integrating factor $\exp \left\{-\int_{0}^{u}[1-\gamma(z)]^{-1}\left[v_{\delta}^{\prime}(z) / v_{\delta}(z)\right] d z\right\}$, one observes that

$$
\begin{aligned}
\frac{d}{d u} & {\left[\exp \left\{-\int_{0}^{u} \frac{1}{1-\gamma(z)} \frac{v_{\delta}^{\prime}(z)}{v_{\delta}(z)} d z\right\} \phi_{\Gamma}(u)\right] } \\
& =\frac{1}{1-\gamma(u)} \exp \left\{-\int_{0}^{u} \frac{1}{1-\gamma(z)} \frac{v_{\delta}^{\prime}(z)}{v_{\delta}(z)} d z\right\}\left[m_{0}^{\prime}(u)-\frac{v_{\delta}^{\prime}(u)}{v_{\delta}(u)} m_{0}(u)\right] w_{*}(u)
\end{aligned}
$$

By noting that

$$
\exp \left\{-\int_{0}^{\infty} \frac{1}{1-\gamma(z)} \frac{v_{\delta}^{\prime}(z)}{v_{\delta}(z)} d z\right\} \leq \exp \left\{-\int_{0}^{\infty} \frac{v_{\delta}^{\prime}(z)}{v_{\delta}(z)} d z\right\}=\frac{1}{v_{\delta}(\infty)} \leq 1
$$

and assuming $\lim _{u \rightarrow \infty} \phi_{\Gamma}(u)=0$, a substitution of $u$ by $x$ in Eq. 2.17 followed by integrating the resulting equation over $x$ from $u$ to $\infty$ leads to

$$
\begin{aligned}
\phi_{\Gamma}(u)= & -\int_{u}^{\infty} \frac{1}{1-\gamma(x)} \exp \left\{-\int_{u}^{x} \frac{1}{1-\gamma(z)} \frac{v_{\delta}^{\prime}(z)}{v_{\delta}(z)} d z\right\} \\
& \times\left[m_{0}^{\prime}(x)-\frac{v_{\delta}^{\prime}(x)}{v_{\delta}(x)} m_{0}(x)\right] w_{*}(x) d x, \quad u \geq 0 .
\end{aligned}
$$

Note that Eq. 2.18 expresses $\phi_{\Gamma}(u)$ in terms of the 'original' Gerber-Shiu function $m_{0}(u)$ without tax payments together with the function $v_{\delta}(u)$, which are both known in a number of risk models. The details regarding the calculations of the quantities $m_{0}(u)$ and $v_{\delta}(u)$ in some risk models which are special cases of the process $\{U(t)\}_{t \geq 0}$ without tax are given in several examples following Remark 2.

Remark 2 Although the differential equation (2.7) for $\phi_{\Gamma}(u)$ involves the function $l(u ; b)$, its solution (2.18) does not. Indeed, the function $l(u ; b)$ has been eliminated from Eq. 2.7 through the use of Eq. 2.15. Nonetheless, the quantity $l(u ; b)$ is also of independent interest and will be used in Section 4. It can be represented as

$$
l(u ; b)=m_{0}(u)-\chi_{\delta}(u ; b) m_{0}(b), \quad 0 \leq u \leq b .
$$

Equation 2.19 can easily be proved probabilistically as follows. Since $l(u ; b)$ is the 'original' Gerber-Shiu function without tax when the surplus process does not upcross level $b$ before ruin, it is simply the 'original' Gerber-Shiu function $m_{0}(u)$ minus the contribution to $m_{0}(u)$ from the sample paths of the surplus process upcrossing level $b$ at least once before ruin (i.e. $\left.\chi_{\delta}(u ; b) m_{0}(b)\right)$. 
Example 1 (Constant premium rate) In the simplest model with constant premium rate $c(.) \equiv c$, the solution $m_{0}(u)$ is given by Gerber and Shiu (1998, Eq. (2.35)), while $v_{\delta}(u)$ can be found in Lin et al. (2003, Section 4).

Example 2 (Multi-threshold risk model) In a multi-threshold risk model (e.g. Lin and Sendova 2008) with $n$ thresholds $\left\{b_{i}\right\}_{i=1}^{n}\left(0<b_{1}<b_{2}<\ldots<b_{n}<\infty\right)$, it is assumed that when the surplus is between levels $b_{i-1}$ and $b_{i}$, the incoming premium rate (net of any dividends) is $c_{i}$ for $i=1,2, \ldots, n+1$ with $b_{0}=0$ and $b_{n+1}=\infty$. This is a special case of the risk model described in Section 1 where $c(x)=c_{i}$ for $b_{i-1} \leq x<b_{i}$. Theorem 4.1 and Corollary 4.3 of Lin and Sendova (2008) provide recursive schemes to evaluate $v_{\delta}(u)$ and $m_{0}(u)$ respectively.

Example 3 (Risk model with credit interest and liquid reserves) In this risk model, it is assumed that the insurer collects premium at a rate $c$. Whenever the surplus level is below a fixed threshold level $\Delta$, the surplus is kept as liquid reserves and does not earn interest. On the other hand, whenever the surplus level exceeds $\Delta$, the excess of the surplus over $\Delta$ earns interest at a rate $\beta>0$ (see Cai et al. 2009). In our context, we set $c(x)=c$ for $x \leq \Delta$ and $c(x)=\beta(x-\Delta)+c$ for $x>\Delta$. Cai et al. (2009) provides an expression for $m_{0}(u)$ via the solution of a Volterra integral equation. The quantity $v_{\delta}(u)$ can be identified in the same manner. The special case $\Delta=0$ corresponds to the risk model with credit interest only; an analysis of $m_{0}(u)$ and $v_{\delta}(u)$ can be found in Cai and Dickson (2002) and Yuen et al. (2007) respectively.

In the next subsection, we shall examine how in general the Gerber-Shiu function $\phi_{\Gamma}(u)$ can be expressed in terms of the 'original' Gerber-Shiu function under a dividend barrier strategy.

\subsection{Relationship of $\phi_{\Gamma}(u)$ with a Dividend Barrier Strategy}

To obtain further insights probabilistically, for a moment we consider the surplus process (1.1) under a dividend barrier strategy at level $b>0$ (see e.g. Lin et al. 2003). Under such a dividend strategy, it is assumed that the entire premium rate is paid as a dividend rate whenever the surplus process reaches level $b$ until the time of the next claim. The resulting surplus process can be viewed as a special case of the tax model $\left\{U_{\Gamma}(t)\right\}_{t \geq 0}$ with

$$
\gamma(x)=\left\{\begin{array}{ll}
0, & 0 \leq x<b \\
1, & x \geq b
\end{array} .\right.
$$

For $0 \leq u \leq b$, let $m(u ; b)$ be the 'original' Gerber-Shiu function under a barrier strategy with initial surplus $u$ and barrier level $b$. From Gerber et al. (2006a), it is known that the so-called dividends-penalty identity

$$
m(u ; b)=m_{0}(u)-V(u ; b) m_{0}^{\prime}(b), \quad 0 \leq u \leq b,
$$

holds where

$$
V(u ; b)=\frac{v_{\delta}(u)}{v_{\delta}^{\prime}(b)}, \quad 0 \leq u \leq b,
$$

is the expected discounted dividend payments before ruin. 
Now we return to the Gerber-Shiu function $\phi_{\Gamma}(u)$ under the general tax structure. Using Eqs. 2.20 and 2.21, Eq. 2.18 can be rewritten as

$$
\begin{aligned}
\phi_{\Gamma}(u)= & \int_{u}^{\infty} \frac{1}{1-\gamma(x)} \exp \left\{-\int_{u}^{x} \frac{1}{1-\gamma(z)} \frac{v_{\delta}^{\prime}(z)}{v_{\delta}(z)} d z\right\} \\
& \times \frac{v_{\delta}^{\prime}(x)}{v_{\delta}(x)}\left[m_{0}(x)-\frac{v_{\delta}(x)}{v_{\delta}^{\prime}(x)} m_{0}^{\prime}(x)\right] w_{*}(x) d x \\
= & -\int_{u}^{\infty} w_{*}(x) m(x ; x) d_{x}\left[\exp \left\{-\int_{u}^{x} \frac{1}{1-\gamma(z)} \frac{v_{\delta}^{\prime}(z)}{v_{\delta}(z)} d z\right\}\right],
\end{aligned}
$$

for $u \geq 0$ where $d_{x}$ is used to specify that the differential is taken w.r.t. $x$.

Remark 3 When $\delta=0$ and $w(.,.) \equiv w_{*}(.) \equiv 1, \phi_{\Gamma}(u)$ reduces to the ruin probability $\psi_{\Gamma}(u)$ in the tax risk model and $m(x ; x)$ becomes the ruin probability in the risk model with a dividend barrier strategy which is known to be 1 . Hence, Eq. 2.22 becomes

$$
\begin{aligned}
\psi_{\Gamma}(u) & =1-\exp \left\{-\int_{u}^{\infty} \frac{1}{1-\gamma(z)} \frac{v_{0}^{\prime}(z)}{v_{0}(z)} d z\right\} \\
& =1-\exp \left\{-\int_{u}^{\infty} \frac{1}{1-\gamma(z)} \frac{d}{d z} \ln v_{0}(z) d z\right\} \\
& =1-\exp \left\{-\int_{u}^{\infty} \frac{1}{1-\gamma(z)} \frac{d}{d z} \ln \frac{v_{0}(z)}{v_{0}(\infty)} d z\right\},
\end{aligned}
$$

for $u \geq 0$. From Eqs. 2.3 and 2.11, it is immediate that $v_{0}(z) / v_{0}(\infty)=1-\psi_{0}(z)$ which together with Eq. 2.23 yields Eq. 1.4. Hence, Eq. 2.22 is an extension of the tax identity (1.4).

Define $\tau_{\Gamma, b}=\inf \left\{t \geq 0: U_{\Gamma}(t)=b\right\}$ to be the first passage time to level $b$ for the tax surplus process $\left\{U_{\Gamma}(t)\right\}_{t \geq 0}$ having Laplace transform

$$
\chi_{\delta, \Gamma}(u ; b)=E\left[e^{-\delta \tau_{\Gamma, b}} I\left(\tau_{\Gamma, b}<T_{\Gamma}\right) \mid U_{\Gamma}(0)=u\right], \quad 0 \leq u \leq b .
$$

Relying on a similar series of arguments as used to derive Eq. 2.18, one can show that

$$
\chi_{\delta, \Gamma}(u ; b)=\exp \left\{-\int_{u}^{b} \frac{1}{1-\gamma(z)} \frac{v_{\delta}^{\prime}(z)}{v_{\delta}(z)} d z\right\}, \quad 0 \leq u \leq b .
$$

We remark that in the case of a constant tax rate $\gamma(.) \equiv \gamma$, it is immediate from Eq. 2.24 (and Eq. 2.11) that

$$
\chi_{\delta, \gamma}(u ; b)=\left[\chi_{\delta}(u ; b)\right]^{\frac{1}{1-\gamma}}, \quad 0 \leq u \leq b .
$$


Formulas (2.24) and (2.25) were obtained by Kyprianou and Zhou (2009) and Albrecher et al. (2008b) respectively as fluctuation identities in the context of a Lévy insurance risk process. Substituting Eq. 2.24 into Eq. 2.22, one easily finds that

$$
\phi_{\Gamma}(u)=-\int_{u}^{\infty} w_{*}(x) m(x ; x) d_{x}\left[\chi_{\delta, \Gamma}(u ; x)\right], \quad u \geq 0 .
$$

Equation 2.26 is a simple identity relating the generalized Gerber-Shiu function in a risk model with tax to the 'original' Gerber-Shiu function in a risk model without tax but with a dividend barrier strategy.

For the rest of this section, we assume $\delta=0$ and further examine Eq. 2.26. For an initial surplus of $U_{\Gamma}(0)=u$ in the tax model, the maximum surplus before ruin is less than $x$ if and only if the surplus process $\left\{U_{\Gamma}(t)\right\}_{t \geq 0}$ drops below 0 before reaching level $x$. Therefore,

$$
\operatorname{Pr}\left\{\bar{U}_{\Gamma}\left(T_{\Gamma}\right)<x, T_{\Gamma}<\infty \mid U_{\Gamma}(0)=u\right\}=1-\chi_{0, \Gamma}(u ; x), \quad 0 \leq u<x,
$$

and hence the (defective) density of $\bar{U}_{\Gamma}\left(T_{\Gamma}\right)$ (with the event that ruin occurs) given $U_{\Gamma}(0)=u$ is

$$
g_{\bar{U}_{\Gamma}\left(T_{\Gamma}\right)}(x \mid u)=-\frac{d}{d x} \chi_{0, \Gamma}(u ; x), \quad 0 \leq u<x .
$$

Using Eq. 2.27, Eq. 2.26 (with $\delta=0$ ) can be written as

$$
\phi_{\Gamma}(u)=\int_{u}^{\infty} w_{*}(x) m(x ; x) g_{\bar{U}_{\Gamma}\left(T_{\Gamma}\right)}(x \mid u) d x, \quad u \geq 0 .
$$

Also, by conditioning $m(x ; x)$ on its first departure from level $x$ and on whether the surplus process under a barrier strategy first reaches level 0 or level $x$ thereafter, we have

$$
m(x ; x)=\left[\int_{0}^{x} \chi_{0}(x-y ; x) p(y) d y\right] m(x ; x)+\int_{0}^{x} l(x-y ; x) p(y) d y+\alpha(x),
$$

or equivalently

$$
\begin{aligned}
m(x ; x) & =\frac{\int_{0}^{x} l(x-y ; x) p(y) d y+\alpha(x)}{1-\int_{0}^{x} \chi_{0}(x-y ; x) p(y) d y} \\
& =\frac{\int_{0}^{x} l(x-y ; x) p(y) d y+\alpha(x)}{\int_{0}^{x}\left[1-\chi_{0}(x-y ; x)\right] p(y) d y+\int_{x}^{\infty} p(y) d y} \\
& =\frac{E\left[E\left[w\left(U_{0}\left(T_{0}^{-}\right),\left|U_{0}\left(T_{0}\right)\right|\right) I\left(T_{0}<\tau_{x}\right) \mid U_{0}(0)=x-Y\right]\right]}{E\left[E\left[I\left(T_{0}<\tau_{x}\right) \mid U_{0}(0)=x-Y\right]\right]}
\end{aligned}
$$

where $Y$ is the generic random variable representing the claim size. 
With the help of Eq. 2.29, a probabilistic interpretation of Eq. 2.28 can be given. Indeed, in Eq. 2.28 the Gerber-Shiu function $\phi_{\Gamma}(u)$ has been conditioned on the maximum surplus level before ruin. If the tax process $\left\{U_{\Gamma}(t)\right\}_{t \geq 0}$ has a maximum surplus before ruin of $x(x \geq u)$,

- $\quad$ a penalty of $w_{*}(x)$ is applied;

- a claim shall occur as soon as $\left\{U_{\Gamma}(t)\right\}_{t \geq 0}$ reaches level $x$. This departure from level $x$ shall be followed by ruin before $\left\{U_{\Gamma}(t)\right\}_{t \geq 0}$ reaches level $x$ (for the maximum surplus to be $x$ ), and is therefore independent of the tax rate. The evaluation of the penalty function $w$ w.r.t. the surplus prior to ruin and the deficit at ruin under the above conditions is given by the right-hand side of Eq. 2.29 which is known to be $m(x ; x)$.

\section{Moments of the Discounted Tax Payments}

By conditioning on the time and the amount of the first claim as well as on whether the process will return to the surplus level just prior to the first claim (if ruin does not occur on the first claim), the quantity $W_{n, \Gamma}(u)$ defined by Eq. 1.8 satisfies

$$
\begin{aligned}
W_{n, \Gamma}(u)= & \int_{0}^{\infty} \lambda e^{-\lambda t} \xi_{n}(u, t) d t+\sum_{j=1}^{n}\left(\begin{array}{c}
n \\
j
\end{array}\right) \int_{0}^{\infty} \lambda e^{-(\lambda+j \delta) t} \xi_{n-j}(u, t) \\
& \times\left[\int_{0}^{f_{\Gamma}(u, t)} \chi_{j \delta}\left(f_{\Gamma}(u, t)-y ; f_{\Gamma}(u, t)\right) p(y) d y\right] W_{j, \Gamma}\left(f_{\Gamma}(u, t)\right) d t,
\end{aligned}
$$

for $u \geq 0$ where

$$
\xi_{j}(u, t)=\left[\int_{0}^{t} e^{-\delta v} c\left(f_{\Gamma}(u, v)\right) \gamma\left(f_{\Gamma}(u, v)\right) d v\right]^{j},
$$

corresponds to the $j$-th moment of the discounted tax payments made from time 0 to the time of the first claim $t$. Letting

$$
\sigma_{j, n}(u, t)=\left(\begin{array}{c}
n \\
j
\end{array}\right) e^{-j \delta t} \xi_{n-j}(u, t),
$$

for $j=0,1, \ldots, n$, a change of the variable of integration from $t$ to $x=f_{\Gamma}(u, t)$ in Eq. 3.1 results in

$$
\begin{aligned}
W_{n, \Gamma}(u)= & \int_{u}^{\infty} \frac{\lambda e^{-\lambda k_{\Gamma}(u, x)}}{c(x)[1-\gamma(x)]} \sigma_{0, n}\left(u, k_{\Gamma}(u, x)\right) d x \\
& +\int_{u}^{\infty} \frac{\lambda e^{-\lambda k_{\Gamma}(u, x)}}{c(x)[1-\gamma(x)]} \sum_{j=1}^{n} \sigma_{j, n}\left(u, k_{\Gamma}(u, x)\right) \\
& \times\left[\int_{0}^{x} \chi_{j \delta}(x-y ; x) p(y) d y\right] W_{j, \Gamma}(x) d x .
\end{aligned}
$$


Differentiating Eq. 3.4 w.r.t. $u$, one finds

$$
\begin{aligned}
W_{n, \Gamma}^{\prime}(u)= & \frac{\lambda}{c(u)[1-\gamma(u)]}\left[1-\int_{0}^{u} \chi_{n \delta}(u-y ; u) p(y) d y\right] W_{n, \Gamma}(u) \\
& +\int_{u}^{\infty} \frac{\lambda e^{-\lambda k_{\Gamma}(u, x)}}{c(x)[1-\gamma(x)]}\left[\frac{\partial}{\partial u} \sigma_{0, n}\left(u, k_{\Gamma}(u, x)\right)\right] d x \\
& +\int_{u}^{\infty} \frac{\lambda e^{-\lambda k_{\Gamma}(u, x)}}{c(x)[1-\gamma(x)]} \sum_{j=1}^{n}\left[\frac{\partial}{\partial u} \sigma_{j, n}\left(u, k_{\Gamma}(u, x)\right)\right] \\
& \times\left[\int_{0}^{x} \chi_{j \delta}(x-y ; x) p(y) d y\right] W_{j, \Gamma}(x) d x,
\end{aligned}
$$

for $u \geq 0$.

Similarly, a change of variable in Eq. 3.2 from $v$ to $z=f_{\Gamma}(u, v)$ results in

$$
\xi_{j}(u, t)=\left[\int_{u}^{f_{\Gamma}(u, t)} e^{-\delta k_{\Gamma}(u, z)} \frac{\gamma(z)}{1-\gamma(z)} d z\right]^{j} .
$$

From Eqs. 3.2 and 3.3, it follows that

$$
\begin{aligned}
\frac{\partial}{\partial u} \sigma_{j, n}\left(u, k_{\Gamma}(u, x)\right)= & \frac{\partial}{\partial u}\left\{\left(\begin{array}{c}
n \\
j
\end{array}\right) e^{-j \delta k_{\Gamma}(u, x)}\left[\int_{u}^{x} e^{-\delta k_{\Gamma}(u, z)} \frac{\gamma(z)}{1-\gamma(z)} d z\right]^{n-j}\right\} \\
= & \frac{j \delta}{c(u)[1-\gamma(u)]} \sigma_{j, n}\left(u, k_{\Gamma}(u, x)\right) \\
& +\frac{(n-j) \delta}{c(u)[1-\gamma(u)]} \sigma_{j, n}\left(u, k_{\Gamma}(u, x)\right) \\
& -\frac{\gamma(u)}{1-\gamma(u)}\left(\begin{array}{c}
n \\
j
\end{array}\right) e^{-j \delta k_{\Gamma}(u, x)}(n-j) \xi_{n-j-1}\left(u, k_{\Gamma}(u, x)\right) \\
= & \frac{n \delta}{c(u)[1-\gamma(u)]} \sigma_{j, n}\left(u, k_{\Gamma}(u, x)\right)-\frac{n \gamma(u)}{1-\gamma(u)} \sigma_{j, n-1}\left(u, k_{\Gamma}(u, x)\right),
\end{aligned}
$$

where $\sigma_{j, n}(u, t)=0$ for $j>n$. Substituting Eq. 3.6 into Eq. 3.5 and then by making use of Eq. 3.4, one finds that

$$
\begin{aligned}
W_{n, \Gamma}^{\prime}(u)= & \frac{1}{1-\gamma(u)}\left[\frac{\lambda+n \delta}{c(u)}-\frac{\lambda}{c(u)} \int_{0}^{u} \chi_{n \delta}(u-y ; u) p(y) d y\right] W_{n, \Gamma}(u) \\
& -\frac{n \gamma(u)}{1-\gamma(u)} W_{n-1, \Gamma}(u),
\end{aligned}
$$

Equation 3.7 together with Eqs. 2.11 and 2.12 leads to

$$
W_{n, \Gamma}^{\prime}(u)=\frac{1}{1-\gamma(u)} \frac{v_{n \delta}^{\prime}(u)}{v_{n \delta}(u)} W_{n, \Gamma}(u)-\frac{n \gamma(u)}{1-\gamma(u)} W_{n-1, \Gamma}(u),
$$

for $u \geq 0$. 
In what follows, we solve the differential equation (3.8) assuming that $\lim _{u \rightarrow \infty} W_{n, \Gamma}(u)$ is finite. Note that this condition is satisfied for most premium rate functions of interest in ruin theory (for instance, when $c($.$) is a bounded function)$ under $\delta>0$. Now, multiplying Eq. 3.8 by the same integrating factor (with $\delta$ replaced by $n \delta$ ) used to obtain Eq. 2.18 yields

$$
W_{n, \Gamma}(u)=\int_{u}^{\infty} \frac{n \gamma(x)}{1-\gamma(x)} \exp \left\{-\int_{u}^{x} \frac{1}{1-\gamma(z)} \frac{v_{n \delta}^{\prime}(z)}{v_{n \delta}(z)} d z\right\} W_{n-1, \Gamma}(x) d x, \quad u \geq 0 .
$$

Using Eq. 2.24, Eq. 3.9 becomes

$$
W_{n, \Gamma}(u)=\int_{u}^{\infty} \frac{n \gamma(x)}{1-\gamma(x)} \chi_{n \delta, \Gamma}(u ; x) W_{n-1, \Gamma}(x) d x, \quad u \geq 0 .
$$

As shown in Renaud (2009, Theorem 3.1), an equation of the form (3.10) also holds true in a Lévy insurance risk model.

Remark 4 In particular, when $n=1$, Eqs. 3.9 and 3.10 become

$$
W_{1, \Gamma}(u)=\int_{u}^{\infty} \frac{\gamma(x)}{1-\gamma(x)} \exp \left\{-\int_{u}^{x} \frac{1}{1-\gamma(z)} \frac{1}{V(z ; z)} d z\right\} d x,
$$

and

$$
W_{1, \Gamma}(u)=\int_{u}^{\infty} \frac{\gamma(x)}{1-\gamma(x)} \chi_{\delta, \Gamma}(u ; x) d x,
$$

respectively, where Eq. 2.21 has been used to obtain Eq. 3.11. Note that Eq. 3.11 relates the expected discounted tax payments to the expected discounted dividends under a barrier strategy, an observation made by Albrecher and Hipp (2007) when $\gamma(.) \equiv \gamma$ and $c(.) \equiv c$. Furthermore, Eq. 3.12 allows for the same probabilistic interpretation as Remark 3.4 of Albrecher et al. (2008b).

\section{Delayed Start of Tax Payments and the Optimal Tax Threshold}

In this section, we pay special attention to the case where tax payments do not start immediately. More precisely, we assume that there is a threshold level $b>0$ (which is no less than the initial surplus $u$ ) such that tax payments start if and when the surplus process reaches level $b$ before ruin. In this section, a predetermined tax structure $\gamma($.) such that $0<\gamma(x)<1$ for $\forall x \geq 0$ is assumed. Note that this predetermined tax structure $\gamma($.) does not allow the tax rate $\gamma(x)$ to be equal to 0 for any $x$; otherwise it may result in $\gamma(b)=0$ which means that tax payments do not actually start at level $b$.

For a threshold tax strategy at level $b$, the actual tax structure is given by

$$
\gamma_{b}(x)=\left\{\begin{array}{ll}
0, & 0 \leq x<b \\
\gamma(x), & x \geq b
\end{array} .\right.
$$

The surplus process under the above tax structure is denoted by $\left\{U_{\Gamma_{b}}(t)\right\}_{t \geq 0}$. We remark that if the process $\left\{U_{\Gamma_{b}}(t)\right\}_{t \geq 0}$ starts with surplus $U_{\Gamma_{b}}(0)=b$, it is equivalent to the process $\left\{U_{\Gamma}(t)\right\}_{t \geq 0}$. 


\subsection{The Gerber-Shiu Function}

Let $\phi_{\Gamma_{b}}(u ; b)$ be the Gerber-Shiu function (1.7) under the tax structure $\gamma_{b}$ (.) defined by Eq. 4.1. Clearly, $\phi_{\Gamma_{b}}(b ; b)=\phi_{\Gamma}(b)$. Applying the tax structure $\gamma_{b}($.$) to Eq. 2.24,$ we also have

$$
\chi_{\delta, \Gamma_{b}}(u ; x)=\chi_{\delta}(u ; x), \quad 0 \leq u \leq x \leq b,
$$

and

$$
\chi_{\delta, \Gamma_{b}}(u ; x)=\chi_{\delta}(u ; b) \chi_{\delta, \Gamma}(b ; x), \quad 0 \leq u \leq b \leq x .
$$

From Eq. 2.26, one deduces that

$$
\begin{aligned}
\phi_{\Gamma_{b}}(u ; b) & =-\int_{u}^{b} w_{*}(x) m(x ; x) d_{x}\left[\chi_{\delta}(u ; x)\right]-\int_{b}^{\infty} w_{*}(x) m(x ; x) d_{x}\left[\chi_{\delta}(u ; b) \chi_{\delta, \Gamma}(b ; x)\right] \\
& =\varphi(u ; b)+\chi_{\delta}(u ; b) \phi_{\Gamma}(b),
\end{aligned}
$$

for $0 \leq u \leq b$, where

$$
\varphi(u ; b)=-\int_{u}^{b} w_{*}(x) m(x ; x) d_{x}\left[\chi_{\delta}(u ; x)\right] .
$$

It can be shown that

$\varphi(u ; b)=E\left[e^{-\delta T_{0}} w\left(U_{0}\left(T_{0}^{-}\right),\left|U_{0}\left(T_{0}\right)\right|\right) w_{*}\left(Z_{T_{0}}\right) I\left(T_{0}<\tau_{b}\right) \mid U_{0}(0)=u\right], \quad 0 \leq u \leq b$,

has solution given by the right-hand side of Eq. 4.3. As a direct consequence, the term $\chi_{\delta}(u ; b) \phi_{\Gamma}(b)$ in Eq. 4.2 represents the contribution to $\phi_{\Gamma_{b}}(u ; b)$ from the sample paths of the surplus process $\left\{U_{\Gamma_{b}}(t)\right\}_{t \geq 0}$ which upcross level $b$ before ruin. It is also instructive to note that $l(u ; b)$ defined in Eq. 2.4 is a special case of $\varphi(u ; b)$ in Eq. 4.4 with $w_{*}(.) \equiv 1$.

Remark 5 We remark that an equation of the form (4.2) also holds true for the generalized Gerber-Shiu function $\phi(u ; b)$ under a dividend barrier strategy at level $b$. By observing that the maximum surplus level before ruin is $b$ almost surely if the surplus process hits level $b$ before ruin, one concludes that

$$
\phi(u ; b)=\varphi(u ; b)+\chi_{\delta}(u ; b) w_{*}(b) m(b ; b), \quad 0 \leq u \leq b .
$$

\subsection{Optimal Threshold Level: Dickson-Waters Modification}

In Albrecher and Hipp (2007), the optimal threshold level which maximizes the expected discounted tax payments before ruin was considered when $\gamma(.) \equiv \gamma$ and $c(.) \equiv c$ under Eq. 4.1. The authors showed that the optimal threshold level is independent of the initial surplus and identified some conditions for the existence of this optimal threshold level. Inspired by Dickson and Waters (2004) and Gerber et al. (2006a), we aim here at maximizing the function

$$
\eta_{\Gamma_{b}}(u ; b)=W_{1, \Gamma_{b}}(u ; b)-m_{\Gamma_{b}}(u ; b), \quad 0 \leq u \leq b,
$$


w.r.t. $b$ for the more general surplus process $\left\{U_{\Gamma_{b}}(t)\right\}_{t \geq 0}$ under the tax structure $\gamma_{b}($.$) ,$ where $m_{\Gamma_{b}}(u ; b)$ is a special case of $\phi_{\Gamma_{b}}(u ; b)$ with $w_{*}(.) \equiv 1$. We remark that the maximization problem (4.5) under $w(x, y)=y$ was first proposed by Dickson and Waters (2004), and was later referred to as the Dickson-Waters modification by Gerber et al. (2006b). This maximization problem was later generalized by Gerber et al. (2006a) to allow for an arbitrary penalty function $w(.,$.$) , and Cheung and$ Drekic (2008) refer to this as the Gerber-Lin-Yang modification.

Since no tax is payable until the surplus process $\left\{U_{\Gamma_{b}}(t)\right\}_{t \geq 0}$ hits level $b$ for the first time (avoiding ruin in the interim), one has

$$
W_{1, \Gamma_{b}}(u ; b)=\chi_{\delta}(u ; b) W_{1, \Gamma}(b), \quad 0 \leq u \leq b .
$$

Substituting Eq. 4.2 (with $w_{*}(.) \equiv 1$ ) and Eq. 4.6 into Eq. 4.5 followed by an application of Eq. 2.19 leads to

$$
\begin{aligned}
\eta_{\Gamma_{b}}(u ; b) & =\chi_{\delta}(u ; b) W_{1, \Gamma}(b)-\left[l(u ; b)+\chi_{\delta}(u ; b) m_{\Gamma}(b)\right] \\
& =\chi_{\delta}(u ; b) W_{1, \Gamma}(b)-\left[m_{0}(u)-\chi_{\delta}(u ; b) m_{0}(b)+\chi_{\delta}(u ; b) m_{\Gamma}(b)\right] \\
& =\chi_{\delta}(u ; b)\left[W_{1, \Gamma}(b)-m_{\Gamma}(b)+m_{0}(b)\right]-m_{0}(u),
\end{aligned}
$$

for $0 \leq u \leq b$. Using Eq. 2.11, Eq. 4.7 can be rewritten as

$$
\eta_{\Gamma_{b}}(u ; b)=v_{\delta}(u) \varpi_{\Gamma}(b)-m_{0}(u), \quad 0 \leq u \leq b,
$$

where

$$
\varpi_{\Gamma}(b)=\frac{W_{1, \Gamma}(b)-m_{\Gamma}(b)+m_{0}(b)}{v_{\delta}(b)} .
$$

In order to identify the optimal threshold level, it is clear from Eq. 4.8 that maximizing $\eta_{\Gamma_{b}}(u ; b)$ w.r.t. $b$ (for a given $u \geq 0$ ) comes down to maximizing $\varpi_{\Gamma}(b)$ on $b \geq u$. Hence, let $b^{*}$ be such that $\varpi_{\Gamma}(b) \leq \varpi_{\Gamma}\left(b^{*}\right)$ for all $b \geq 0$. Then, for any $0 \leq u \leq b^{*}$, the optimal threshold level that maximizes $\eta_{\Gamma_{b}}(u ; b)$ w.r.t. $b$ is $b^{*}$ which is independent of the initial surplus level $u$.

A necessary condition for a positive (i.e. non-zero) $b^{*}$ to maximize $\varpi_{\Gamma}(b)$ on $b \geq 0$ is

$$
\varpi_{\Gamma}^{\prime}\left(b^{*}\right)=0 .
$$

Using Eq. 4.9, the condition (4.10) becomes

$$
\frac{v_{\delta}^{\prime}\left(b^{*}\right)}{v_{\delta}\left(b^{*}\right)}=\frac{W_{1, \Gamma}^{\prime}\left(b^{*}\right)-m_{\Gamma}^{\prime}\left(b^{*}\right)+m_{0}^{\prime}\left(b^{*}\right)}{W_{1, \Gamma}\left(b^{*}\right)-m_{\Gamma}\left(b^{*}\right)+m_{0}\left(b^{*}\right)} .
$$

From Eqs. 2.16 and 3.8, we also have that

$$
m_{\Gamma}^{\prime}(b)=\frac{1}{1-\gamma(b)} \frac{v_{\delta}^{\prime}(b)}{v_{\delta}(b)} m_{\Gamma}(b)+\frac{1}{1-\gamma(b)}\left[m_{0}^{\prime}(b)-\frac{v_{\delta}^{\prime}(b)}{v_{\delta}(b)} m_{0}(b)\right],
$$

and

$$
W_{1, \Gamma}^{\prime}(b)=\frac{1}{1-\gamma(b)} \frac{v_{\delta}^{\prime}(b)}{v_{\delta}(b)} W_{1, \Gamma}(b)-\frac{\gamma(b)}{1-\gamma(b)},
$$


respectively. Capitalizing on Eqs. 4.12 and 4.13, one easily sees that

$$
\begin{aligned}
W_{1, \Gamma}^{\prime}(b)-m_{\Gamma}^{\prime}(b)+m_{0}^{\prime}(b)= & \frac{1}{1-\gamma(b)} \frac{v_{\delta}^{\prime}(b)}{v_{\delta}(b)}\left[W_{1, \Gamma}(b)-m_{\Gamma}(b)+m_{0}(b)\right] \\
& -\frac{\gamma(b)}{1-\gamma(b)}\left[1+m_{0}^{\prime}(b)\right],
\end{aligned}
$$

and therefore

$$
\frac{W_{1, \Gamma}^{\prime}(b)-m_{\Gamma}^{\prime}(b)+m_{0}^{\prime}(b)}{W_{1, \Gamma}(b)-m_{\Gamma}(b)+m_{0}(b)}=\frac{1}{1-\gamma(b)} \frac{v_{\delta}^{\prime}(b)}{v_{\delta}(b)}-\frac{\gamma(b)}{1-\gamma(b)} \frac{1+m_{0}^{\prime}(b)}{W_{1, \Gamma}(b)-m_{\Gamma}(b)+m_{0}(b)} .
$$

By Gerber et al. (2006a, equation (11)), we know that

$$
1+m_{0}^{\prime}(b)=\frac{v_{\delta}^{\prime}(b)}{v_{\delta}(b)}\left[V(b ; b)-m(b ; b)+m_{0}(b)\right] .
$$

Upon substitution of Eq. 4.15 into Eq. 4.14, one deduces from Eq. 4.11 that the necessary condition for a positive $b^{*}$ to maximize $\varpi_{\Gamma}(b)$ is given by

$$
\frac{V\left(b^{*} ; b^{*}\right)-m\left(b^{*} ; b^{*}\right)+m_{0}\left(b^{*}\right)}{W_{1, \Gamma}\left(b^{*}\right)-m_{\Gamma}\left(b^{*}\right)+m_{0}\left(b^{*}\right)}=1,
$$

or equivalently

$$
W_{1, \Gamma}\left(b^{*}\right)-m_{\Gamma}\left(b^{*}\right)=V\left(b^{*} ; b^{*}\right)-m\left(b^{*} ; b^{*}\right)
$$

Interestingly, using Eqs. 4.12 and 4.13, a positive $b^{*}$ satisfying Eq. 4.16 also implies that

$$
\begin{aligned}
W_{1, \Gamma}^{\prime}\left(b^{*}\right)-m_{\Gamma}^{\prime}\left(b^{*}\right)= & \frac{1}{1-\gamma\left(b^{*}\right)} \frac{v_{\delta}^{\prime}\left(b^{*}\right)}{v_{\delta}\left(b^{*}\right)}\left[W_{1, \Gamma}\left(b^{*}\right)-m_{\Gamma}\left(b^{*}\right)\right]-\frac{\gamma\left(b^{*}\right)}{1-\gamma\left(b^{*}\right)} \\
& -\frac{1}{1-\gamma\left(b^{*}\right)}\left[m_{0}^{\prime}\left(b^{*}\right)-\frac{v_{\delta}^{\prime}\left(b^{*}\right)}{v_{\delta}\left(b^{*}\right)} m_{0}\left(b^{*}\right)\right] \\
= & \frac{1}{1-\gamma\left(b^{*}\right)} \frac{1}{V\left(b^{*} ; b^{*}\right)}\left[V\left(b^{*} ; b^{*}\right)-m\left(b^{*} ; b^{*}\right)\right] \\
& -\frac{\gamma\left(b^{*}\right)}{1-\gamma\left(b^{*}\right)}+\frac{1}{1-\gamma\left(b^{*}\right)} \frac{1}{V\left(b^{*} ; b^{*}\right)} m\left(b^{*} ; b^{*}\right) \\
= & 1,
\end{aligned}
$$

where the second last line follows from Eqs. 2.20 and 2.21. Note that the conditions (4.16) and (4.17) reduce to equations (18) and (16) in Albrecher and Hipp (2007) 
respectively when $w(.,.) \equiv 0$. Furthermore, by utilizing the expression (4.5), the condition (4.17) is indeed equivalent to

$$
\left.\frac{d}{d b} \eta_{\Gamma_{b}}(b ; b)\right|_{b=b^{*}}=1,
$$

which says that if tax is to be collected immediately (i.e. $u=b$ ), then at the optimal tax threshold $b^{*}$ the marginal gain in the value function $\eta_{\Gamma_{b}}(b ; b)$ is identical to the marginal increase in capital injection.

Although the necessary condition (4.17) (or equivalently (4.18)) is mathematically interesting and allows for economic interpretation, unfortunately in general we do not expect to be able to find explicit expression for the optimal value $b^{*}$. This is indeed already evident in the most simplest case where both tax rate and premium rate are constant and claims follow exponential distribution (see Albrecher and Hipp 2007, Example 1). In that case, $W_{1, \gamma}(u)$ is expressed in terms of a Gauss hypergeometric series and an explicit formula for $b^{*}$ is unlikely to be available.

\section{Concluding Remarks}

In the context of the compound Poisson risk model with surplus-dependent premium rate and general tax structure, this paper extends the tax identity to a generalized Gerber-Shiu function involving the maximum surplus prior to ruin. Moments of the discounted tax payments before ruin and the optimal tax threshold level are also studied.

Some of the results in the present model such as the special case (Eq. 1.4) of the general tax identity (Eq. 2.26), the Laplace transform of the first passage time (Eq. 2.24) and the moments of discounted tax payments (Eq. 3.10) have an identical counterpart in the Lévy insurance risk model with general tax structure (see Kyprianou and Zhou 2009; Renaud 2009). It is worth pointing out that the model considered in this paper is more general in terms of the premium income with a surplus-dependent premium rate. However, the mathematical derivations of these results in these two classes of risk models are quite different. This raises the natural question as to whether the afore-mentioned results hold true in a more general class of risk models.

Acknowledgements The authors would like to thank the anonymous referee for helpful comments and suggestions which improve the presentation of the paper. Support for Eric C.K. Cheung from a start-up fund provided by the Faculty of Science and the Department of Statistics and Actuarial Science at the University of Hong Kong and support for David Landriault from the Natural Sciences and Engineering Research Council of Canada are gratefully acknowledged.

Open Access This article is distributed under the terms of the Creative Commons Attribution Noncommercial License which permits any noncommercial use, distribution, and reproduction in any medium, provided the original author(s) and source are credited.

\section{References}

Albrecher H, Hipp C (2007) Lundberg's risk process with tax. Blätter der DGVFM 28(1):13-28

Albrecher H, Badescu AL, Landriault D (2008a) On the dual risk model with tax payments. Insur Math Econ 42(3):1086-1094 
Albrecher H, Renaud J-F, Zhou X (2008b) A Lévy insurance risk process with tax. J Appl Probab 45(2):363-375

Albrecher H, Borst S, Boxma O, Resing J (2009) The tax identity in risk theory-a simple proof and an extension. Insur Math Econ 44(2):304-306

Biffis E, Kyprianou AE (2010) A note on scale functions and the time value of ruin for Lévy insurance risk processes. Insur Math Econ 46(1):85-91

Cai J (2007) On the time value of absolute ruin with debit interest. Adv Appl Probab 39(2):343-359

Cai J, Dickson DCM (2002) On the expected discounted penalty function at ruin of a surplus process with interest. Insur Math Econ 30(3):389-404

Cai J, Feng R, Willmot GE (2009) The compound Poisson surplus model with interest and liquid reserves: analysis of the Gerber-Shiu discounted penalty function. Methodol Comput Appl Probab 11(3):401-423

Cheung ECK, Drekic S (2008) Dividend moments in the dual risk model: exact and approximate approaches. ASTIN Bull 38(2):399-422

Cheung ECK, Landriault D (2010) A generalized penalty function with the maximum surplus prior to ruin in a MAP risk model. Insur Math Econ 46(1):127-134

Dickson DCM, Waters HR (2004) Some optimal dividends problems. ASTIN Bull 34(1):49-74

Gerber HU, Lin SX, Yang H (2006a) A note on the dividends-penalty identity and the optimal dividend barrier. ASTIN Bull 36(2):489-503

Gerber HU, Shiu ESW, Smith N (2006b) Maximizing dividends without bankruptcy. ASTIN Bull 36(1):5-23

Gerber HU, Shiu ESW (1998) On the time value of ruin. N Am Actuar J 2(1):48-72

Kyprianou AE, Zhou X (2009) General tax structures and the Lévy insurance risk model. J Appl Probab 46(4):1146-1156

Lakshmikantham V, Rao RMM (1995) Theory of integro-differential equations. Gordon and Breach Science Publishers, Lausanne

Lin SX, Pavlova KP (2006) The compound Poisson risk model with a threshold dividend strategy. Insur Math Econ 38(1):57-80

Lin SX, Sendova KP (2008) The compound Poisson risk model with multiple thresholds. Insur Math Econ 42(2):617-627

Lin SX, Willmot GE, Drekic S (2003) The classical risk model with a constant dividend barrier: analysis of the Gerber-Shiu discounted penalty function. Insur Math Econ 33(3):551-566

Ming R-X, Wang W-Y, Xiao L-Q (2010) On the time value of absolute ruin with tax. Insur Math Econ 46(1):67-84

Renaud J-F (2009) The distribution of tax payments in a Lévy insurance risk model with a surplusdependent taxation structure. Insur Math Econ 45(2):242-246

Yuen KC, Wang G, Li WK (2007) The Gerber-Shiu expected discounted penalty function for risk processes with interest and a constant dividend barrier. Insur Math Econ 40(1):104-112

Wei L (2009) Ruin probability in the presence of interest earnings and tax payments. Insur Math Econ 45(1):133-138 